\title{
Characteristics of Vibration of Reinforced Concrete Buildings of Different Ages
}

\author{
Gegentana ${ }^{1}$,Narenmandula ${ }^{1}$, Hongge Wang ${ }^{1}$, Jianzhong $\mathrm{Bao}^{2}$, Fengxiang $\mathrm{Li}^{2}$, \\ KAWASE Hiroshi ${ }^{3}$, MATUSHIMA Youyichi ${ }^{3}$ \\ ${ }^{1}$ College of Geographical Science,Inner Mongolia Normal University, Natural Disaster \\ Prevention ResearchInstitute, Hohhot,010022,China, \\ ${ }^{2}$ Inner Mongolia Urban Plannig\&Municipal Engineering Design Research Institute, \\ Hohhot,010070, China, \\ ${ }^{3}$ Disaster Prevention Research Institute, Kyoto University, Kyoto, 611001, Japan
}

\section{不同年代钢筋混凝土建筑物的振动特性研究}

\author{
格根塔娜 ${ }^{1}$, 那仁满都拉 ${ }^{1}$, 王鸿䳜 ${ }^{1}$, 包健众 ${ }^{2}$, 李风祥 ${ }^{2}$, 川濑博 ${ }^{3}$, 松岛信一 ${ }^{3}$ \\ ${ }^{1}$ 内蒙古师范大学地理科学学院, 内蒙古师范大学自然灾害防治研究所, \\ 呼和浩特, 010022, 中国, \\ ${ }^{2}$ 内蒙古城市规划市政设计研究院, 呼和浩特, 010070, 中国, \\ ${ }^{3}$ 京都大学防灾研究所, 611001, 日本京都,
}

\begin{abstract}
In order to determine the vibration characteristics of reinforced concrete buildings of different ages, shapes similar to Hohhot, the same height reinforced concrete building built in different years as the research object, the use of micro-observation methods often mastered the natural cycle of the building. The natural cycle of the building's correlation analysis found that the natural period of the construction of the building's longer have gradually longer trend. Preliminary analysis concluded that the main reason may be the building itself naturally weathered old building leading to the stiffness (or seismic endurance) decreased, while the impact strength of the building material changes brought is not obvious.
\end{abstract}

Key words: Vibration; Different years; Always jog observation; RC

\section{摘要}

为了掌握不同年代钢筋混凝土建筑物的振动 特性, 以呼和浩特市区不同年代建造的形状类 似、同等高度的钢筋混凝土建筑物作为研究对 象, 采用常时微动观测方法获得了建筑物的固 有周期。对固有周期与建筑年代相关关系分析, 掌握建造年代越久建筑物的固有周期有逐渐 变长的趋势。初步分析推断, 该趋势的主要原 因可能是建筑物本身的自然风化老旧导致建 筑刚度 (或抗震耐力) 逐渐降低, 而建筑材料 的强度变化所带来的影响不为明显。

关键字: 振动特性; 不同年代; 常时微动观测; 钢筋混凝土

\section{1. 引言}

中国是一个地震活动较频繁的国家, 我国 大陆不但处于环太平洋地震带和欧亚地震带 之间, 还有印度板块和太平洋板块从南面和东 南面的挤压, 这种特殊的构造环境使我国成为 了一个地震活动频繁的国家。据统计, 中国仅 占全球陆地面积的 $1 / 15$, 但是大陆地震却占 
Risk Analysis and Crisis Response in Big Data Era (RAC-16)

全球的 $1 / 3$, 全国 $60 \%$ 的国土、 $50 \%$ 的城市、 $67 \%$ 的大城市位于烈度 7 度及以上烈度区之 内 $^{[1,2]}$ 。我国毕竟还是发展中国家, 建筑物的 抗震能力、地震灾害预测技术及防御技术与发 展国家相比还存在一定的差距。中国中西部 欠发达地区是印度板块运动的最大受力区, 也是板内地震最活跃, 最大受害区, 至此, 我 国中西部欠发达地区将面对的自然灾害挑战 会十分严峻, 发生破坏性地震的可能性很高, 这将对我国可持续发展造成一定负面影响 ${ }^{[1-4]}$ 。

改革开放以来, 随着社会和经济的高速发 展和城市用地的紧张, 钢筋混泥土建筑物的兴 建愈发猛烈。可是唐山、汶川和玉树等地震灾 害告诉我们, 建筑物抗震能力低是造成重大人 员伤亡和经济损失的主要原因 ${ }^{[5]}$ 。一般钢筋混 凝土结构的经济寿命为 60 年, 住宅的自然寿 命一般要大于经济寿命, 随着年代的变迁, 建 筑抗震能力逐渐减少, 会导致出现各种安全隐 患, 加上未来可能发生的地震灾害等不可避免 的情况, 因此, 掌握现有建筑物的抗震能力或 抗震能力变化特征是非常重要的任务。目前推 测建筑抗震能力一般使用建筑设计材料为基 准, 但是在建筑使用过程中抗震耐力会出现变 化。为了掌握建筑物的现有抗震能力, 使用常 时微动观测方法的研究在逐渐增多 ${ }^{[6-8]}$ 。常时 微动观测技术主要应用在地基土划分、地震烈 度增量计算、建筑物抗震性能评价、工程场地 地震安全评价、滑坡的地质调查等方面, 为工 程地质以及震害预测等提供基础性资料 ${ }^{[8-10]}$ 。 吴志坚等人通过对汶川地震的震害调查验证 了常时微动观测结果与震后实际情况有一致

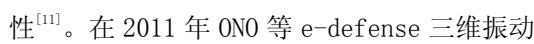
台试验结果显示常时微动观测方法能很好地 反映足尺钢框架结构的损伤程度和刚度 ${ }^{[12]}$ 。可 是, 目前建筑抗震能力随着建筑年代的变化而 变化的研究极少, 影响着城市建设和城市管理 中防灾减灾政策的导入和实践等。如果掌握建 筑物抗震能力随着年代变化而变化的特征, 再 结合建筑设计材料来推测建筑物当前和未来 的抗震能力, 可以为城市防灾规划和城市地震 安全评估等工作提供科学性依据。

本文为了间接推测建筑抗震能力随着建 造年代变化而变化的特征, 采用常时微动观测 方法, 以中西部欠发达地区呼和浩特市区为研
究对象, 分析形状类似、高度同等、建造年代 不同的钢筋混凝土建筑物的固有周期, 掌握了 不同年代建筑物的固有周期随着年代变化而 变化特性, 并初步提出建筑物固有周期的建筑 年代变化原因要素。

\section{2. 观测建筑概况及其观测数据分析方法}

\section{1 观测建筑概况及测量步骤}

那仁满都拉等在钢筋混凝土建筑物振动 特性研究中证实了建筑物的固有周期与建筑 物的高度、形状、年代、用途有着密切关系 ${ }^{[9]}$ 。 因此本文为了避免建筑物的高度及形状对建 筑物固有周期产生影响, 选择了呼和浩特市区 内形状、建造年代不同的 6 层、8 层、11 层和 12 层等几组钢筋混凝土建筑物。表 1 显示对 象建筑物的概况。

表 1 观测建筑物的基本信息及固有周期 序 建筑 楼 建筑建筑 建筑 建筑 顶层/1层 号编号层年代 $\left(\mathrm{m}^{2}\right)$ 形状用途短轴EW 长轴NS

\begin{tabular}{|c|c|c|c|c|c|c|c|c|}
\hline | & HSND01 & 6 & 2011 & 938 & 长方 & 住宅 & 0.30 & 0.27 \\
\hline 2 & HSNDO2 & 6 & 2009 & 516 & 长方 & 住宅 & 0.26 & 0.27 \\
\hline 3 & HSND03 & 6 & 1997 & 936 & 长方 & 商业 & 0.29 & 0.38 \\
\hline 4 & HSNDO4 & 6 & 2014 & 598 & 长方 & 住宅 & 0.26 & 0.24 \\
\hline 5 & HSND05 & 6 & 2014 & 760 & 长方 & 住宅 & 0.26 & 0.30 \\
\hline 6 & HSND06 & 6 & 2006 & 1062 & 长方 & 教育 & 0.41 & 0.41 \\
\hline 7 & HSND07 & 6 & 2012 & 891 & 长方 & 住宅 & 0.27 & 0.26 \\
\hline 8 & HSND08 & 6 & 2011 & 2546 & 长方 & 教育 & 0.33 & 0.33 \\
\hline 9 & HSND09 & 6 & 2011 & 4508 & 长方 & 教育 & 0.37 & 0.35 \\
\hline ) & HSND10 & 6 & 2010 & 3828 & 长方 & 教育 & 0.51 & 0.42 \\
\hline 11 & HSND11 & 6 & 2000 & 5842 & 长方 & 商业 & 0.52 & 0.51 \\
\hline 12 & HSND12 & 6 & 2005 & 704 & 长方 & 教育 & 0.36 & 0.36 \\
\hline 13 & HSND13 & 6 & 2009 & 616 & 长方 & 住宅 & 0.31 & 0.30 \\
\hline 4 & HSND14 & 6 & 2012 & 1736 & 长方 & 住宅 & 0.27 & 0.24 \\
\hline 15 & HSND15 & 6 & 2013 & 455 & 长方 & 住宅 & 0.25 & 0.27 \\
\hline 16 & HSND16 & 6 & 2007 & 1200 & 长方 & 写字 & 0.23 & 0.33 \\
\hline 17 & HSND17 & 8 & 1995 & 630 & 长方 & 行政 & 0.49 & 0.36 \\
\hline 18 & 3 HSND18 & 8 & 1992 & 861 & 长方 & 商业 & 0.47 & 0.44 \\
\hline 19 & HSND19 & 8 & 1985 & 1200 & 长方 & 商业 & 0.45 & 0.49 \\
\hline 20 & HSND20 & 8 & 2013 & 576 & 长方 & 住宅 & 0.31 & 0.46 \\
\hline 21 & HSND21 & 8 & 2007 & 690 & 长方 & 医疗 & 0.53 & 0.49 \\
\hline 22 & HSND22 & 8 & 2008 & 660 & 长方 & 住宅 & 0.34 & 0.38 \\
\hline 23 & HSND23 & 8 & 1990 & 648 & 长方 & 商业 & 0.41 & 0.51 \\
\hline 24 & HSND24 & 8 & 2003 & 980 & 长方 & 商业 & 0.40 & 0.42 \\
\hline 25 & HSND25 & 8 & 2008 & 810 & 长方 & 住宅 & 0.43 & 0.43 \\
\hline 26 & HSND26 & 8 & 1987 & 672 & 长方 & 教育 & 0.48 & 0.41 \\
\hline 27 & HSND27 & 8 & 1999 & 280 & 长方 & 教育 & 0.44 & 0.48 \\
\hline 28 & 3 HSND28 & 8 & 2001 & 1800 & 长方 & 行政 & 0.56 & 0.40 \\
\hline 29 & HSND29 & 11 & 2013 & 308 & 长方 & 住宅 & 0.54 & 0.56 \\
\hline & HSND30 & 11 & 2011 & 854 & 长方 & 准 & 0.54 & 0.59 \\
\hline
\end{tabular}




\section{Risk Analysis and Crisis Response in Big Data Era (RAC-16)}

31 HSND31 112005289 正方 住宅 $0.56 \quad 0.62$ 32 HSND32 $112011 \quad 1040$ 长方 住宅 $0.55 \quad 0.45$ 33 HSND33 112004944 长方 住宅 $0.52 \quad 0.52$ 34 HSND34 112009588 长方 住宅 $0.47 \quad 0.40$ 35 HSND35 $112009 \quad 770$ 长方 住宅 $0.53 \quad 0.51$ $\begin{array}{lllllll}36 & \text { HSND36 } 112011 & 767 & \text { 长方 住宅 } & 0.49 & 0.41\end{array}$

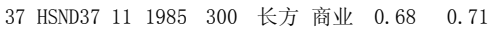
$\begin{array}{llllllll}38 & \text { HSND38 } & 11 & 1986 & 527 & \text { 长方 写字 } & 0.67 & 0.64\end{array}$ 39 HSND39 $112014 \quad 957$ 长方 住宅 $0.46 \quad 0.36$ 40 HSND40 $112008 \quad 1320$ 长方 商业 $0.68 \quad 0.70$ 41 HSND41 $11 \quad 1998 \quad 2200$ 长方 教育 $0.65 \quad 0.52$ 42 HSND42 $112010 \quad 559$ 长方 住宅 $0.48 \quad 0.46$ 43 HSND43 $112010 \quad 936$ 长方 住宅 $0.60 \quad 0.61$ 44 HSND44 $112013 \quad 406$ 长方 住宅 $0.42 \quad 0.43$ 45 HSND45 112011336 长方 住宅 $0.47 \quad 0.41$ 46 HSND46 1120101053 长方 住宅 $0.50 \quad 0.37$ 47 HSND47 $112002 \quad 468$ 长方 住宅 $0.58 \quad 0.42$ 48 HSND48 $112009 \quad 495$ 长方 住宅 $0.45 \quad 0.36$ 49 HSND49 $112004 \quad 1050$ 长方 住宅 $0.58 \quad 0.51$

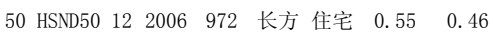

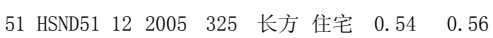
$\begin{array}{lllllll}52 & \text { HSND52 } 12 & 2004 & 891 & \text { 长方 写字 } & 0.63 & 0.60\end{array}$ 53 HSND53 $122013 \quad 806$ 长方 住宅 $0.48 \quad 0.62$ 54 HSND54 $12 \quad 2010 \quad 336$ 长方 住宅 $0.55 \quad 0.61$ 55 HSND55 $122007 \quad 1652$ 长方 住宅 $0.61 \quad 0.56$ $\begin{array}{lllllll}56 & \text { HSND56 } 121999 & 1480 & \text { 长方 写字 } & 0.58 & 0.76\end{array}$ $\begin{array}{lllllll}57 \text { HSND57 } 122002 & 1584 & \text { 长方 写字 } & 0.58 & 0.58\end{array}$ $\begin{array}{lllllll}58 & \text { HSND58 } 12 & 2002 & 1562 & \text { 长方 写字 } & 0.72 & 0.61\end{array}$ $\begin{array}{llllll}59 & \text { HSND59 } 122009 & 1364 & \text { 长方 写字 } & 0.68 & 0.67\end{array}$

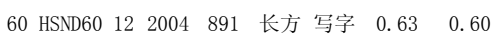
61 HSND61 $12 \quad 2000 \quad 814$ 长方 行政 $0.56 \quad 0.54$

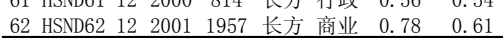

本次研究采用了日本应用地质公司制造 的常时微动仪, 该仪器的 3 个传感器定制在仪 器内部一个可以调平的钢板上面, 可以测到两 个水平方向 (NS 和 EW 方向) 和一个垂直方向 (UD 方向) 的加速度成分, 采样频率为 $200 \mathrm{~Hz}$ 。 观测在白天进行, 3 台常时微动仪器分别放置 在建筑物的 1 层 (1F)、顶层 (R) 和外面的自 由地面 (G), 图 1 显示常时微动仪观测时的位 置图。仪器放置的 NS 和 EW 方向需和建筑物的 长轴和短轴方向相对应, 一层和顶层的仪器要 尽量相互垂直, 并靠近建筑物的承重墙或柱子 等主要结构。选定外面的自由地面的位置时要 注意避免地窝、管道以及地下车库等空地, 需 放置在离建筑物 3 米以外的自然地面上。此外, 需要用 GPS 控制时间的准确性, 3 个仪器必须 从同一个时间点开始测量, 保证数据的同步性。 每栋建筑物观测用时 $15 \min \times 2$, 中间间隔 1
到 2 分钟。需要注意的是在观测过程当中围绕 仪器一米之内要避免有人为活动, 因为常时微 动是一种没有震源的振幅很小的微弱振动, 为 了数据的精准要保持安静无动态的观测环境。

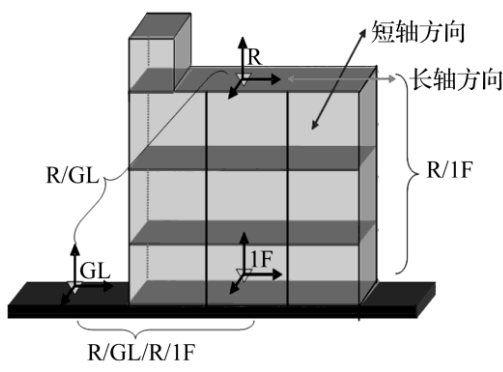

图 1 常时微动仪的位置图

为了更好的掌握建筑物振动特征, 采用常 时微动仪观测的同时采集了建筑物的面积、建 造年代、结构、层数、形状、用途、平面和立 面图等基本信息, 并对观测建筑物拍照留存。 建筑物所在经纬度使用便捷 GPS 来定位。

\section{2 观测数据的处理与分析}

首先, 把观测得到的 30 分钟的数据采用 搭接 $50 \%$ 的观测数据叠加方法, 切出时间带为 81.92 秒的数据。然后从 22 个时间带中选择 出没有受干扰的数据进行快速傅立叶变换计 算得出它的傅立叶频谱, 会出现 NS、EW 和 UD 方向的傅立叶频谱。再计算不同方向的建筑物 顶层和 1 层傅立叶频谱比。从 NS 方向和 $\mathrm{EW}$ 方 向傅立叶频谱比中读取第一个高峰值, 作为建 筑物的长轴 (NS) 和短轴 (EW) 的第一自振周 期, 即建筑物固有周期。

\section{3. 不同年代钢筋混凝土结构建筑物振动特性}

按上述常时微动观测数据分析法, 分别记 算出建筑物的顶层和 1 层的第一次固有周期, 表 1 列出建筑物的固有周期值。

图 2 至图 5 显示 6 层、8 层、11 层和 12 层建筑物固有周期的建筑年代变化趋势。图中 横轴代表建筑物建造的年代, 竖轴代表建筑物 
Risk Analysis and Crisis Response in Big Data Era (RAC-16)

的固有周期; 菱形的图例代表建筑物短轴的固 有周期, 代表公式为图中公式 $\mathrm{y}$, 圆形图例代 表建筑物长轴的固有周期, 代表公式为图中公 式 $\mathrm{w}$; 直线代表建筑物短轴的固有周期走势, 虚线代表建筑物长轴的固有周期走势。从图中 可以看出, 同层建筑物随着年代的推移, 不论 短轴还是长轴, 建筑建造的年代越久, 它的固 有周期有变长的趋势。从公式 $\mathrm{T}=2 \Pi(\mathrm{M} / \mathrm{K})$ 得 知, 在质量 (M) 同等的情况下, 建筑物的刚 度 $(\mathrm{K})$ 和固有周期 $(\mathrm{T})$ 呈反比例关系 ${ }^{[8]}$ 。因 此, 推测建造年代越久建筑物的刚度或抗震而 力就越低, 固有周期则越长。另外, 因为对象 建筑物的数量过少, 没能以建筑用途进行分类 研究, 不同用途的钢筋混凝土建筑物的振动特 性或许可能存在差异, 在这方面尚需更进一步 的分析和研究。

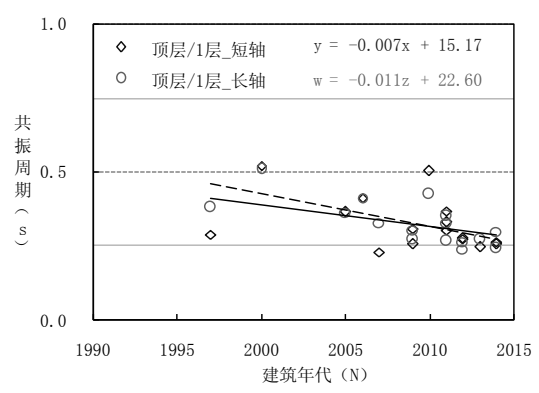

图 26 层建筑物固有周期的建筑年代变化

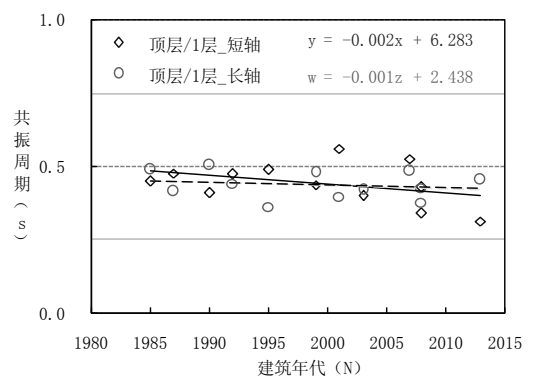

图 38 层建筑物固有周期的建筑年代变化

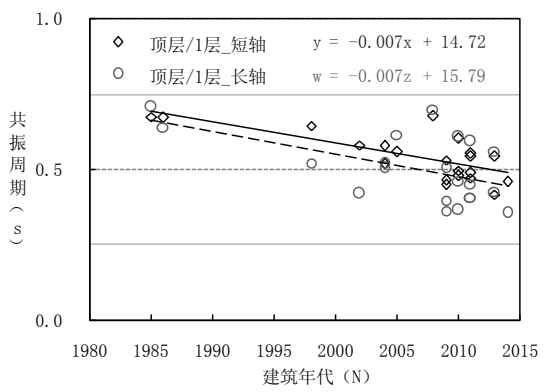

图 $4 \quad 11$ 层建筑物固有周期的建筑年代变化

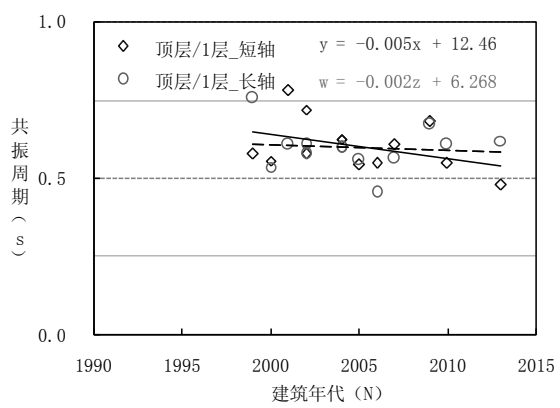

图 512 层建筑物固有周期的建筑年代变化

\section{4. 建筑物固有周期的建筑年代变化原因分析}

影响建筑物固有周期变长的原因有很多 方面, 主要以建筑物当地的自然条件引起的风 化老旧、建筑混凝土材料特性变化、建筑结构 变化、建筑施工情况和建筑后期保护维修等原 因为主。本文中选用的建筑物为结构相同和形 状类似的钢筋混凝土框架结构, 并且建筑物都 分布在呼和浩特市区, 建筑施工技术和后期管 理水平也基本相同, 所以可以认为对象建筑物 固有周期变化的主要影响原因为建筑物本身 的风化老旧和钢筋混凝土材料的进化发展两 个要素。

\section{1 建筑材料变化对固有周期的影响}

为了分析建筑材料对建筑固有周期的影 响, 对比《混凝土结构设计规范》GB50010-2002 和《混凝土结构设计规范》GB50010-2010 发 
Risk Analysis and Crisis Response in Big Data Era (RAC-16)

现, 2010 年以后钢筋混凝土强度等级得到很 大提升。新规范明确规定钢筋混凝土结构的混 凝土强度等级不应低于 $\mathrm{C} 20$, 采用高强度级别 的钢筋时, 混凝土强度等级还应增加, 钢筋也 向高强、高性能方向发展, 淘汰低强度的钢筋, 形成 300、400、500Mpa 强度梯次 ${ }^{[13-14]}$ 。因此, 以《混凝土结构设计规范》GB50010-2010 颁 布时间为标准，考虑到建筑施工时间以 2011 年为界限比较建筑物的固有周期, 间接推测混 凝土和钢筋的强度提升对建筑振动特性的影 响。因为观测数据的限制, 进一步分析了 6 层 和 11 层建筑两组数据, 图 6 和图 7 显示 2011 年前后建的建筑物固有周期及其平均趋势线 的比较结果。从图中可以看出, 2011 年之后 的建筑物的固有周期趋势线整体偏低与 2011 年之前的建筑物的固有周期趋势线。该趋势可 能反映着混凝土强度等级和钢筋强度提升所 引起的建筑固有周期变化情况。但无论 2011 年前建的或者后建的建筑物的固有周期随着 建筑建造年代的变化而变长, 因此, 可以初步 推断为钢筋混凝土的强度提升对建筑固有周 期随着建造年代变化而变长的趋势有影响, 但 不是主要影响因素。

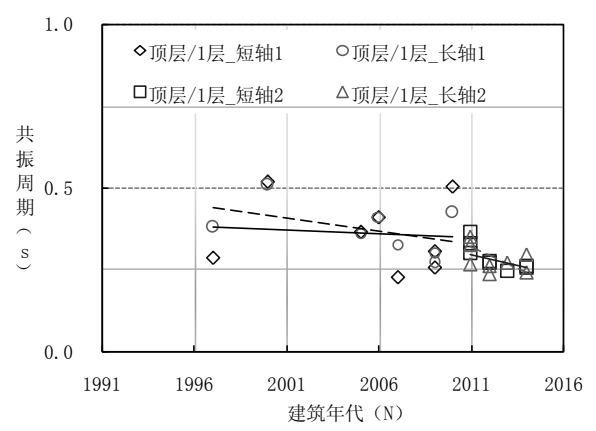

图 66 层建筑物固有周期的建筑年代变化

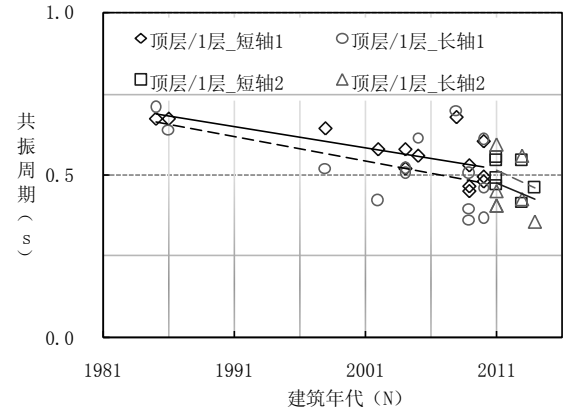

图 $7 \quad 11$ 层建筑物固有周期的建筑年代变化

\section{2 建筑物本身的风化老旧对固有周期的影} 响

通过对钢筋混凝材料风化特征分析发现， 钢筋混凝结构建筑本身由于时间长了会出现 一些缺陷 ${ }^{[15-16]}$ 。随着建筑物的使用, 首先, 由 于建筑物表面混凝土受到大气、水汽或各种腐 蚀性介质的作用产生一系列化学反应, 使混凝 土结构开裂、膨胀、溶解受到破坏, 进而使混 凝土强度急剧降低。其次, 由于混凝土保护层 的破坏深度达到钢筋表面时, 钢筋也受到大气、 水汽和各种腐蚀性介质的接触, 导致钢筋强度 也逐步降低, 而且钢筋表面的锈蚀产物使钢筋 体积变大, 对混凝土保护层产生应力, 从而导 致混凝土产生进一步破坏。在如此风化老旧的 循环作用下, 导致整体钢筋混凝土建筑的刚度 和抗震耐力降低, 使建筑固有周期变长。

以上结论, 初步推断建筑物的自然风化老 旧对建筑物的刚度和抗震耐力的影响较大, 因 此, 从观测数据中得到建造年代越久的钢筋混 凝土建筑物的固有周期有逐渐变长的趋势。

\section{5. 总结}

本文以呼和浩特市区不同年代建造的形 状相似、同等高度的钢筋混凝土建筑物作为研 究对象, 采用常时微动观测方法, 掌握了建筑 物随着年代变化的固有周期的变化特征。通过 分析 6 层、8 层、11 层和 12 层等四组建筑物 的固有周期发现, 得知建造年代越久建筑物的 固有周期有变长的趋势。初步分析推断, 导致 上述结论的主要原因为建筑物本身的自然风 
化老旧导致建筑刚度或抗震耐力逐渐降低, 而 钢筋混凝土材料的强度变化所带来的影响不 为明显。该建造年代越久建筑物的固有周期有 变长的趋势, 可结合建筑设计材料来推测建筑 物当前抗震能力和未来的抗震能力变化, 为城 市地震安全评估工作等提供科学依据。今后, 增加建筑设计与施工材料、建筑物数量和用途 等详细设置条件, 对钢筋混凝土建筑物的振动 特性进行更进一步详细研究, 掌握建筑物刚度 或抗震能力的建筑年代变化而变化趋势。

\section{Acknowledgements}

This study was supported by National Natural Science Foundation of China (41461101), and The Project Sponsored by the Scientific Research Foundation for the Returned Overseas Chinese Scholars, State Education Ministry,and The introduction of high-level scientific research personnel project start-up funds, Inner Mongolia Normal University( YJRC12005).

\section{致谢}

本研究得到国家自然科学基金项目(41461101), 教育部留学回国人员科研启动基金资助项目, 内蒙古师范大学高层次人才科研启动经费项 目(YJRC12005)的资助。

\section{参考文献}

[1]聂高众, 高建国, 马宗晋. 中国未来 $10 \sim 15$ 年地震灾害的风险评估. 自然灾害学报, 2002, 11 (1) : 68-73.

[2]科技部国家计委国家经贸委灾害综合研究 组, 灾害, 社会, 减灾, 发展. 北京:气象 出版社. 2000.

[3]聂高众, 高建国. 21 世纪中国的自然灾害发 展趋势——以地震和旱涝为例. 第四纪研 究, 2001, 21 (3) :249-261.

[4] J.P. Yan, S.S Li, J. Bai, X.Y. Liu. The spatial symmetry axis of earthquake hazard in China. Journal of Risk Analysis and Crisis Response, 2013, 3 (1) : 59-64.
[5]赵小凡. 浅谈钢筋混凝土结构的发展. 建筑 与工程, 2011, (27): 701.

[6]YAGI Shigehua, TOBITA Jun, FUKUWA Nobuo.Effect of soil-Structure Interaction on Transfer Function Estimation of Low-Rise R.C. Buildings using Microtremor. Journal of Structural Engineering, AIJ, 2000, 46B, 435-444.

[7]HO Narenmandula, KAWASE Hiroshi. Evaluation of Earthquake Response and Dynamic Characteristics of RC and Wooden Buildings in FUKUKA city Obtained from Aftershocks of the West off FUKUKA Earthquake and Microtremor Data. Journal of Structural and Construction Engineering. AIJ, 2006, 605, 63-70.

[8]那仁满都拉. 基于常时微动观测的钢筋混凝 土建筑物振动特性及其地域差异. 振动与 冲击, 2016, 35 (4) :22-27.

[9]陈宏林. 工程地震勘察方法. 地震出版 社. 1998.

[10]布仁. 基于常时微动观测的呼和浩特市区 地基土振动特性及分类研究. 内蒙古师范 大学学报自然版, 2004, 43(1):86-91.

[11]吴志坚, 车爱兰, 王兰民, 等. 常时微动测 试在汶川地震甘肃灾区建筑结构震害调查 中的应用. 西北地震学报, 2009 , $31(1): 87-90$.

[12]Masaru ONO,KenjiKANAZAWA.Seismic Damage Detection of A High-Rise Steel Building on the Full-Scale Shaking Table Test. Journal of Structural and Construction Engineering. AIJ, 2011, 662, 775-783.

[13]《混凝土结构设计规范》 GB50010-2002.

[14]《混凝土结构设计规范》GB50010-2010.

[15]赵国藩. 钢筋混凝土结构发展现状及展望. 中国土木工程学会第八届年会论文 集. 1998.

[16]陈合成, 葛培华, 刘军. 钢筋混凝土的腐蚀 与防护.石油化工腐蚀与防护, 2003,20(6): 10-13. 